\title{
RENAL COLIC IN INFANTS
}

BY

\author{
R. A. GIBBONS, M.D., M.R.C.P.,
}

PHYSICIAN TO THE GROSVENOR HOSPITAL FOR WOMEN AND CHILDREN

Received August 10th, 1895-Read January 14th, 1896.

THE subject of this paper is one of which I can find no notice in the 'Transactions' of this or of any other Society. I therefore hope that what I have written may be deemed worthy of the attention of the Fellows. I have only referred to cases occurring in infants whom I have seen in private practice, and I think it better to relate these cases in the first place, and reserve any remarks until afterwards.

I have selected the following as examples of the subject of this communication.

CaSE 1.-A boy æt. 10 months. Was first seen on December 3rd, 1885, because he was suddenly seized with a screaming fit, and seemed to be in great pain. The least movement evidently gave additional pain, and caused him to scream. He was lying on the arm of the nurse, on his left side, the legs being drawn up, and the thighs flexed on the abdomen. His face was flushed and expressive of terror, especially if anyone came very near him. When allowed to remain quiet in the nurse's arms his pain seemed bearable, for he did not scream. He had vomited several times. 
I heard from his mother and nurse that he had been perfectly well in the morning, and that he was suddenly seized with this attack whilst being amused.

There is nothing of importance to relate regarding the previous history. He has always been apparently healthy. I could not gather that he had suffered from any previous attacks of a similar nature to the one he was then in. He was born at full time, and his mother nursed him until he was between eight and nine months old. His diet when I saw him consisted of boiled cow's milk, water, sugar of milk, and lime water, one ounce to a pint of food, and a heaped teaspoonful of sugar of milk to each bottle. In addition to this he was having two meals a day of Savory and Moore's food. This diet had been ordered some time previously, and had not been altered.

His father is very gouty, and there is a history of syphilis on his side. The mother is gouty, and comes of a gouty stock. He has two sisters and two brothers. The elder brother suffers from asthma of a severe type. The elder sister, aged sixteen, is suffering from what is probably a renal calculus, and I have attended her on three occasions for attacks of great lumbar pain, accompanied by hæmaturia, the latter usually lasting several days. The urine sometimes contains pus cells.

There is no history of phthisis on the side of the mother or father.

The child has been perfectly well since birth, and has caused no anxiety whatever.

I found on examination that the heart and lungs appeared normal. The pulse was frequent and the respiration hurried. The temperature was $102^{\circ}$. On having him gently undressed I noticed that he assumed the position already mentioned. He would allow me, after being present a short time, to touch the abdomen over the left flank, but if the least pressure were made on the right side in front or behind, violent pain was produced. This seemed to be especially well marked 
if the left hand were placed gently over the lumbar region behind, and slight pressure made with the right hand in front, although this manipulation could be tolerated on the opposite side. It was clear from this examination that the region of the right kidney was the seat of pain. The testicle of the right side was drawn up. On looking at the urine it was observed to be perfectly clear, and the nurse stated that it was scanty in amount, and was passed in small quantities and with considerable pain. At the bottom of the vessel a few minute grains, like Cayenne pepper, were visible. A mixture containing some compound tincture of camphor, bromide of ammonium, and carbonate of lithia was prescribed, as well as a warm bath and a linseed meal poultice to the loins. The urine sent to me for examination had a sp. gr. of 1020, contained a small quantity of albumen, no sugar or bile; and, microscopically, free uric acid was found, as well as mucus corpuscles and blood. The crystals of uric acid-lozenge-shaped variety-were not very numerous.

The following morning I learned that although the treatment seemed to have caused amelioration in the urgent symptoms, the child had suffered from many severe paroxysms of pain during the night, and was still in great pain if touched over the region of the right kidney. The medicine was continued, and as the day passed the child seemed to be easier, the intervals between the paroxysms of pain becoming longer. It was now observed that the urine contained a very large quantity of uric acid, and there were many aggregations of crystals held together by mucus. This was easily discernible to the naked eye. On more than one occasion a small piece, less than a quarter of the size of a split pea, of thickish mortar-like material was passed, which proved on examination to consist of uric acid. The next morning, when again I saw the child, I heard that he had passed a disturbed night, but with few paroxysms of pain, and he now appeared much better, although he disliked to be touched on the affected side. The temperature was 
normal. During the day he had hardly any pain, and the water was comparatively free from uric acid. In the evening he seemed well again.

Four months afterwards he had an attack similar in most respects to the foregoing, and three months subsequently, when seventeen months old, another one, whilst between three and four months later he had the last attack. I saw him more than three years afterwards, when he looked perfectly well, and I learned from his mother that he had been troubled with no return.

CASE 2.-A boy æt. 9 months. Was first seen in December, 1886. I found him in exactly the same condition as that just related, except that the left side was affected instead of the right. $\mathrm{He}$ was in acute pain on my arrival, lying on the nurse's arm, on his right side, and having legs and thighs drawn up, and the left testicle retracted. He had vomited frequently. The temperature was $102.4^{\circ}$, the pulse and respiration hurried. He could not bear to be touched, and screamed if moved. There was nothing amiss with the lungs or heart, but it was easily discernible that the least touch anywhere about the region of the left kidney caused increase of pain. The nurse informed me that he constantly passed a little water, apparently with much difficulty and with aggravation of pain. I noticed that there were some crystals of uric acid in it, but the water was perfectly clear. On examination microscopically I observed abundance of uric acid-ordinary lozenge-shaped crystals and masses of rosettes, as well as mucus corpuscles and blood-corpuscles.

The treatment adopted was similar to that of the previous case. The time of my first seeing the child was late in the evening, soon after he had been suddenly seized. During the night he suffered from almost constant pain, but the paroxysms of acute pain became gradually less frequent and less severe; the amount of urine became greater, and a larger amount of uric acid was observable, but in the form of solitary crystals, and of masses aggre- 
gated together in the midst of mucus suspended in the urine. On allowing the urine to stand in a conical glass it was noted that the sp. gr. was 1022, that there was a copious amount of uric acid, and that there were two or three minute but distinct masses, larger than the size of a hemp-seed, which could be broken down with the finger into powder. Blood-corpuscles were also observed.

In twenty-four hours from the onset of the attack the child was much better, and twelve hours later appeared well. He never had another similar attack, although I know that on more than one occasion he passed abundance of free uric acid.

This child had been fed for the first few weeks of life on Swiss milk and cow's milk, but was then changed to a wet-nurse, a strong healthy woman, who was still nursing him at the time of his attack.

In the family history I have to mention that gout is strongly marked both on the father's and on the mother's side. A brother of the child suffered from "sandy water" on many occasions. Three sisters are healthy. There is no history of phthisis on either side, and there is nothing else of importance to note.

Case 3.-A boy æt. 22 months. Was first seen in May, 1886. At the time of my visit he was evidently in acute pain, cried continuously, and shrieked if touched. The legs were drawn up, and the thighs flexed on the abdomen. Neither testicle could be felt. It was impossible to make a proper examination, on account of the increased pain brought on by the slightest touch.

I learned from the nurse that no water had been passed for some hours, but she believed he was anxious to pass some and could not. She could tell me nothing of importance concerning the previous state of the water. The temperature was $101 \frac{1}{2}^{\circ}$, and the pulse rapid. The skin was bathed in a profuse perspiration. I was told by the mother and the nurse that the child was perfectly well until that day, when he was suddenly seized with this acute pain. 
I ordered a warm bath, poultices to the loins, and a mixture containing bromide of ammonium and compound tincture of camphor. A few hours later I saw him, when he appeared somewhat easier, but was still in much pain. I now found that this pain seemed to emanate from the right lumbar region. As in the other cases, the least pressure about the right renal region caused an exacerbation of the symptoms. On auscultation and percussion of the chest, nothing amiss could be detected. As the nurse told me that he had evidently great desire to micturate, and the bladder seemed distended, a small soft French elastic catheter was passed along the urethra without meeting with any obstruction, and a considerable amount of urine drawn off. He seemed somewhat relieved by this, and had no further difficulty in the actual passing of water, although the desire was frequent, and micturition seemed painful.

During the night he had repeated paroxysms of pain, but the intervals between them grew longer. On examination in the morning I found the same tenderness in the right flank, and the recurrence of acute pain on attempting to make pressure. The treatment was continued, and towards the latter part of the day he seemed easier. The temperature, which was $100^{\circ}$ in the morning, became $99^{\circ}$ in the evening. The testicles were now visible.

The following night he had some sleep which seemed refreshing. There was scarcely any pain, and in the morning the temperature was normal. He could not, however, bear any pressure over the renal region, but unless the affected region was touched the pain seemed to have disappeared.

On examination of the urine drawn off by the catheter, it was found to be clear, with a sp. gr. of 1020, and to contain free uric acid. On standing in a conical glass a copious deposit of uric acid crystals, with a considerable amount of mucus, was thrown down. There were masses of the crystals aggregated together, and suspended by mucus. With heating and nitric acid a good trace of 
albumen was found; and under the microscope, blood corpuscles, mucus corpuscles, and abundance of crystals of uric acid-lozenge-shaped crystals, cubes, and large rosettes-as well as renal epithelium and crystals of oxalate of lime.

On examining the urine the following day, blood was still observed microscopically, with masses of uric acid crystals, but no fragment of anything resembling a calculus could be discovered. It appeared to me at first that something might possibly be blocking the urethra before the catheter was passed, seeing that urination was afterwards perfectly free, but nothing was discovered, although directions were given to keep all the water, and spasm of the urethra due to reflex action might, of course, account for the condition of not passing water.

The pain in the lumbar region gradually disappeared, but the urine for three days later contained masses of uric acid suspended in mucus. The albumen disappeared, and likewise the blood.

The family history is important. Gout is strongly marked, both on the father's side and on the mother's. There is one other child, an infant, who is healthy. The little patient was brought up by hand, being fed on cow's milk, and was rather rickety.

Beyond an accident at ten months old, which made him feverish for a few days, owing to a lacerated wound on the hand, there is nothing to report about his previous history. He has always been quite well. He has never had to my knowledge another similar attack, but he was much thinner after the one now described, and was some time in regaining strength, looking anæmic for several weeks. His diet at the time of the attack consisted of milk, biscuits, beef tea, chicken broth, mutton tea, and nursery pudding.

CASE 4.-A boy æt. 23 months. First seen September 7th, 1886, on account of a severe attack of pain and screaming. The child was put to bed as usual, and suddenly awoke 
about 10 p.m. with screaming. 'Nothing could pacify him, and when $I$ arrived he was still screaming, but seemed pale and exhausted. The nurse kept him lying on her lap, but he was restless and could not be kept still. His face and body were covered with perspiration, and the surface of the body was cool. He vomited several times. The pulse was feeble and rapid, but the temperature was below normal, $97^{\circ}$. Beyond the great restlessness there was no difficulty in examining the chest, for the paroxysms of pain appeared to grow less, and the screaming ceased as the child became exhausted. There was nothing wrong with the lungs, and the heart sounds were merely feeble from his general exhaustion. He seemed to prefer lying on his left side with the knees drawn up. I could not palpate the abdomen, for the least touch anywhere seemed to cause great pain, and I believed that the right lumbar region was clearly the most sensitive of all. Neither testicle could be felt. He had been perfectly well until suddenly seized with this pain whilst sleeping. A mixture containing bromide of ammonium, aromatic spirit of ammonia, and compound tincture of camphor was ordered, with hot fomentations to the loins.

The next morning I heard that he had a second attack of pain, lasting a few minutes, about three hours after the first attack, and a third one about 6 in the morning, also lasting a few minutes. When I saw him he was languid, and the pulse was feeble, but he did not seem ill. He was tender over the loins, and especially over the right renal region. The testicles could be felt. The liver and spleen were not palpable. He remained languid for three or four days. The water was clear, sp. gr. 1020 to 1024, with a trace of albumen; no sugar or bile. Several specimens contained abundance of free crystals of uric acid, lozenge-shaped and rosettes, and mucus, also renal epithelium and blood-cells. In the specimen of the night following the attack a small mortar-like mass was found, which could easily be crushed with the finger, and was composed of uric acid. 
The boy's diet before the attack consisted of milk, toast and rusks, milky puddings, and a little meat, chicken, or fish in the middle of the day. Before this attack he has suffered occasionally from eczema of the face and head, but from nothing else. The father is not strong, and has suffered occasionally from abscesses. He is very gouty, and comes of a gouty stock. The mother is extremely gouty, and suffers greatly from megrim and severe eczema of the body, which has proved most obstinate to treatment. There is only an infant brother.

This boy has had several subsequent attacks precisely similar to the above, always coming on without warning, and always accompanied by the passage of large quantities of free uric acid, and twice by the passage of minute mortar-like substances.

CASt 5.-A boy æt. 6 months. First seen February 10th, 1887,-a brother of the above. I was asked to see him, as I happened to be in the house at the moment of seizure. He was apparently well when he was suddenly seized with screaming, and seemed to be in acute pain. Undressing him caused an increase of suffering. There was nothing to be discovered by examination except great tenderness over the left loin. Any pressure there aggravated his suffering. He screamed until he appeared perfectly exhausted. There was no rise of temperature. I noted that the testicles were retracted. $\mathrm{He}$ vomited frequently. When he seemed quieter and could apparently bear a little more examination he did not mind the abdomen being touched. Pressure in the left lumbar region, with one hand in front and the other behind, brought on a sharp paroxysm of pain.

The treatment was the same as for the other cases. The urine, which was difficult to collect, was acid in reaction, clear, and contained a large quantity of free uric acid and a slight trace of albumen, which subsequently disappeared. Microscopically there were bloodcells. The child remained more or less in discomfort all vol. LXXIX. 
day, and had two recurrences of acute pain. There was slight eczema about the scalp at the time of this attack, and three days afterwards he developed a severe acute attack of eczema of the head and face. His diet consisted of cow's milk and water, with lime water and sugar of milk.

From time to time I saw this child, and he had other similar attacks, although none so severe. He frequently, however, passed large quantities of free uric acid without any acute pain being manifest.

A sister of these children was subsequently born; and although she never to my knowledge suffered from any attack like her brothers, she frequently passed considerable quantities of free uric acid.

CASE 6.-A girl æt. 12 months. First seen November 3rd, 1887, because she was suddenly seized with violent pain, screaming, and vomiting. I ascertained that she had been perfectly well, and was quite in her usual health on the morning of seizure. When I arrived she was in a paroxysm of pain and screaming loudly, and was terrified. With great suffering she was undressed. She was well nourished, and nothing could be detected amiss except that she had evidently pain about the loins. She preferred to lie on her right side on the nurse's lap, and could not bear the least touch about the left renal region, but at first examination was impossible, her contortions were so great. Afterwards she became quite exhausted and slightly collapsed, with feeble pulse, depressed temperature, and cool skin.

Similar treatment to that for the others was adopted after the patient had been gently placed in a warm bath, which seemed to give her ease. She was very depressed all day after this attack, which lasted some little time. The urine was quite clear, acid, and had abundance of uric acid. During the following night she had another severe attack, much the same as the first, lasting a considerable time, for the paroxysms of pain returned; and the following day she 
passed several minute concretions of a dark brown colour. The urine also contained a faint trace of albumen; and microscopically some blood-cells were observed, as well as lozenge-shaped crystals of uric acid, and some crystals of oxalate of lime. For the next five days the child remained feeble, and then seemed in her usual health. She never had another attack like the above, but I have often seen her since, and she has frequently passed quantities of free uric acid. She was having milk, beef tea, and chicken broth for diet.

She was an only child. The father is gouty, and comes of a very gouty family. The mother is neurotic and gouty, and has a history of gout in her family for generations back.

Two years afterwards a sister was born, who proved to be a delicate child, liable to bronchial attacks, but the point of interest is that she, too, frequently had attacks of "gravel," but never such an attack as above described. They generally caused irritability, restlessness at night and by day, with general uneasiness about the loins and constant micturition.

When we speak of renal colic we usually understand that the patient is seized with severe pain in one loin, that it comes on very suddenly and with great violence, and frequently after some kind of movement, such as jumping or riding on horseback, or jolting in an uncomfortable carriage. We know that this pain, although starting from the loin and running down in the direction of the ureter, may radiate in all directions, so that sharp pain may be felt in other parts of the abdomen and down the leg. The testicle is usually retracted towards the inguinal canal, and may be extremely sensitive, painful to the touch, and swollen. The patient may be faint, cold, collapsed, covered with a clammy perspiration-in short, in real agony. Occasionally the suffering is so severe that he may be thrown into a convulsion. During an attack the pulse gradually grows frequent, small, and easily com- 
pressible, whilst the respiration is also hurried. At the same time the temperature rises; vomiting is frequently met with from the very commencement of the seizure, and may continue at intervals all through the attack. The pain abates after a time, and the patient may feel comparatively comfortable, when another paroxysm of agony returns, to be followed by temporary relief or entire cessation as the case may be. During the actual seizure, the slightest movement of the body is generally followed by increase of pain, so that the patient remains as still as possible, and may assume curious attitudes in order to cause some mitigation of pain.

Whilst the attack lasts micturition is generally frequent, and the urine is perfectly clear. On the other hand, strangury may be present with bloody urine. If the kidney on the opposite side is healthy, natural urine may be secreted as usual. It must be remarked that although the urine may appear natural to the naked eye, on microscopical examination blood may be discovered, which is frequently of the greatest value in forming a diagnosis in cases simulating gall-stone colic. Cases have been reported where the urine voided has been habitually purulent and bloody from calculous pyelitis, and where, during an impaction of a stone in the ureter, the water has appeared normal, proving that the kidney on the opposite side was healthy (Ebstein, Ziemssen's Cyclopædia, vol. $x v$, p. 717,1877$)$.

The foregoing clinical picture is the one with which we are familiar in dealing with adults, but the cases just related are remarkable as having occurred in young infants.

Now it becomes a matter of importance to note that although the symptoms of renal colic may be present, yet no calculus may be subsequently discovered. When one is passed the matter of diagnosis is absolutely settled, but frequently it happens that this is not so, and there may be considerable doubt about the case. I mention this because, in the cases cited, there was no perfect stone discovered, although there were numerous masses of uric acid crystals. 
Moreover it must also be observed that it is occasionally some days before a stone is passed after cessation of all pain or discomfort. I have a patient, a lady, who occasionally consults me regarding the passage of renal calculi, and whom I have seen in the paroxysm of pain attending the passage of a stone. She has a small box of calculi, the collection of years, of all sizes, from that of a mass one would imagine almost incapable of passing along the ureter, down to that of a mere grain. In her case it has been occasionally days after the attack before she has passed the stone from the bladder, and, what is of great importance for this paper, she has frequently not found the stone at all. On one occasion, when I attended her, no stone was passed subsequent to the attack of renal colic, but on examination of the water I found it loaded with enormous crystals of uric acid, in addition to little masses of crystals held together by mucus, and these were present for several days. There can be no doubt that an impacted stone of certain composition may become disintegrated, and be gradually washed away by the stream of urine. In such an instance it shows how important it is to keep watch upon the state of the urine if there is the least doubt about the diagnosis. And this must be of especial importance in the case of children, for they can tell us nothing of the kind or situation of the pain. It is so common for infants to have attacks of intestinal colic, that unless there is something strikingly severe not much notice is taken of the symptoms. Even in the adult, with every advantage of examination, it may be impossible to say whether an attack of acute pain, such as is usually described as renal colic, is really due to the passage of a gall-stone or renal calculus. I have myself seen one of the ablest clinical physicians we have ever had diagnose renal colic when subsequent signs proved that the attack was due to gall-stones, and it is within my knowledge that such mistakes have not unfrequently happened. Indeed, I have heard Sir William Jenner say that in some cases, when in doubt, the only way to be certain is to wait for a day or 
so. If the case be one of the passage of a gall-stone, jaundice will probably appear, whereas if the pain be due to renal colic albumen will be present, or appear subsequently, in the water. In the cases brought forward blood in the urine was noticed. If then, in the adult, diagnosis between the pain due to renal colic and that due to the passage of a gall-stone be difficult, it is much more so to say in an infant what the paroxysm of pain is due to. In the cases noted it will be observed that there was evidently an increase of the pain if the loin on the affected side were touched, whilst the only position at all tolerable was when the children were lying on the unaffected side on the arm of the nurse. In the first case the slightest touch in the renal region threw the child into a paroxysm of pain, and all the movements necessary in undressing the child for examination, although most gently done, exaggerated the sufferings. It is quite evident, therefore, that the cause of pain was in the lumbar region.

In dealing with this subject it is necessary to state that in all the cases I have seen I have had no reason to confound this pain with that associated with the passage of uric acid crystals. Few practitioners of any observation can have failed to note how frequently the presence of uric acid in the urine is associated with restlessness, screaming, and general disturbance in infants. But here it is common to find sandy material in the urine if it can be saved, or on the napkins. Frequently this is sufficient to cause irritation, redness, or swelling at the orifice of the urethra; not unfrequently, indeed, if phimosis is present, in boys this urine, highly charged with uric acid, may collect beneath the prepuce, and set up balanitis, with considerable œdema of the surrounding tissues. In girls great irritation of the vulva is quite common from the same kind of urine. In association with this disturbance it not unfrequently happens that there is tenderness in both lumbar regions, and dislike to being touched at all. But I have never been struck with the fact that one loin has been markedly more sensitive than the other, although 
such cases are really common, and one has ample opportunities of seeing them. They occur also at varying intervals in the same child, and the passage of uric acid may last from a few hours to several days, when the disturbance ceases. I can speak from my own experience when I say that this disturbance, caused by the occasional presence of uric acid, is most common in the children of gouty parents, and I constantly come across it. In some the passage of free uric acid is almost always present; and at this moment I have a boy aged five under my care, who has suffered for some time now from recurrent attacks of irritation caused by abundant uric acid crystals passed with the urine. This causes more or less incontinence of urine, for he frequently wets himself, both by night and by day. Yet he has no stone which can be detected in the bladder, although he has been sounded by several well-known surgeons, nor has he a stone, as far as one can judge, in the kidneys, and he has never had any attacks of renal colic. His father, as a boy, suffered from exactly the same condition, and has been subject to attacks of gout for years, whilst the father's mother suffered in the same manner. Although this case I mention is an aggravated one, I frequently have under my notice children suffering from the same kind of affection.

There can be no doubt but that the occurrence of true renal colic in infants is exceedingly rare, and yet stone in the bladder is not uncommon. Statistics with regard to the presence of stone in the bladder prove how frequently it occurs ; thus, according to Dr. Prout, out of 1256 patients admitted into the Bristol, Leeds, and Norwich hospitals for lithotomy or lithotrity, 500, or nearly 40 per cent., were under ten years of age.'

In a paper by Mr. Morgan, published in the 74th volume of the Transactions, "On 114 Consecutive Cases of Operation for Calculus in the Bladder performed in the Hospital for Sick Children," twenty-nine are entered as

1 'On the Nature and Treatment of Stomach and Renal Diseases,' 1848, Appendix, Table III, p. 593 
being of the age of three or under, the youngest being sixteen months.

Of ninety-eight calculi in children in the museum of the Royal College of Surgeons of England, kindly tabulated for me by Mr. Morison, the age is mentioned in seventy-nine cases, and of these eighteen were three years of age and under, the youngest being twelve months. In one of these cases it is noted that there were numerous small concretions, and that they were passed by the urethra. In another in the same table a small white calculus was passsed by the urethra.

Henoch says ${ }^{1}$ that stone is just as common in children during the first years of life as in adults. Again, we know how extremely common it is for children to have copious deposits in the urine, with the least disturbance to the general system; and even in new-born children, according to Henoch, ${ }^{2}$ uric acid infarcts are invariably present. Professor Schlossberger, who examined 199 children dying within thirty days from birth, found uric acid in the tubuli uriniferi in 32 per cent. These observations were also confirmed by Professor Martin, of Jena ('Archiv f. physiol. Heilkunde,' vol. ix, and Schmidt's 'Jahrbücher,' December, 1850, p. 333). These are gradually washed out of the tubuli, and the condition found will, therefore, depend upon the date after birth at which the examination is made, for Henoch has noted them as long as seven or eight weeks after birth. It is obvious, therefore, that with such a tendency to the formation of uric acid the chances of a calculus forming are great, whether it be found in the kidney or bladder. Uric acid concretions are the most common. They constitute five sixths of the total number of renal calculi, and wholly or in part the great majority of those found in the bladder ('Practice of Medicine,' Bristowe, 7th edit., p. 846).

1 'Lectures on Children's Diseases,' translated for the New Sydenham Society from the fourth edition (1889) by John Thomson, M.B., F.R.C.P.Edin., vol. ii, p. 175.

2 Op. cit., vol. ii, p. 174. 
Dr. Dickinson, in his work ' On Renal and Urinary Affections ' (vol. iii), has tabulated the composition of ninety-one calculi occurring in the pathological museums of London. Of these fifty-two were simple and thirty-nine compound, and of the latter, twenty-two were composed of two ingredients. Dr. Dickinson remarks that oxalate of lime is the most frequent constituent of compound calculi, existing in about two thirds of the whole number, and that calculi, especially if they be composed of uric acid, oxalate of lime, or cystin, generally take their first concrete form in the pelvis of the kidney.

It may be presumed that the usual manner of formation is that these crystals of uric acid irritate the delicate mucous membrane of the pelvis of the kidney, causing an increase in activity of the cell-growth, and thus a greater amount of secretion,-in fact, a catarrhal condition; this secretion, covering the crystals, causes them to adhere and form little masses, and these masses, joining together and constantly being augmented, become the nucleus of a calculus. If the accumulation formed is washed away before it becomes too large to easily pass from the pelvis along the ureter, no harm is done. If, on the other hand, it cannot be washed down the ureter, it becomes impacted, giving rise to renal colic, and remains in the pelvis of the kidney, to become the source of future trouble and danger.

In the cases brought forward my belief is that the cause of the colic was still soft and friable, and in its very commencement, for under the microscope large masses of crystals of uric acid were discovered with numerous mucus corpuscles as well as blood corpuscles. It therefore appeared to me, so large and numerous were these masses-far larger than I have ever seen in the examination of the urine of infants suffering merely from what may be termed "attacks of uric acid "-that the commencing calculus had not become too hard for being crushed and disintegrated by the combined action of muscular contraction of the ureter and the flow of urine. Of 
course it is possible that some small central, hardened portion or core may have passed and have escaped observation. At any rate, in these cases mentioned I did not discern anything beyond what I have described, although instruction was given to the nurse to keep a careful watch upon the urine. The fact that infants occasionally pass renal calculi is by no means rare, although that they suffer from true renal colic must be a matter of great rarity. In reference to the passage of renal calculi several well-known authorities may be quoted. Henoch mentions a case (op. cit., vol. i, p. 189) of a child five months old, who passed round fragments of the size of a pin's head, which were recognised as uric acid concretions. The case is quoted by him as an instance of reflex irritation starting in the urinary organs, for the child cried always before passing water, and developed first convulsions, and subsequently contractures of the toes of both feet, and of the fingers and kneejoints.

Rilliet and Barthez ('Maladies des Enfants,' p. 720) say that they have never met with a calculus which was impacted in the ureter, and that in their experience calculi are most common in the right kidney, where they are often large, and are rarely met with in both ; but that if in both, the calculi are largely developed. They mention (p. 721) that infants may be attacked by pain in the lumbar region, or in other parts of the urinary organs, and that it may be intense and paroxysmal-a true nephritic colic.

It is remarkable that, in spite of the great tendency in infants and children to uric acid concretions and urinary deposits, so little mention is met with of true renal colic. The only explanation is that the affection must be extremely rare.

Dr. West says ${ }^{1}$ that the formation of lithic acid in the kidneys goes on without giving rise to any very obvious symptoms, and that "he has but rarely seen a

$i$ 'Lectures on the Diseases of Infancy and Childhood,' 6th edition, p. 713. 
child suffer from pain of that severe character which in the adult not infrequently accompanies the descent of a calculus from the kidney to the bladder." Dr. Eustace Smith ('A Practical Treatise on Disease in Children,' 1884, p. 815) remarks that "when the concretion passes from the kidney into the ureter and downward into the bladder there is always pain, but the child suffers far less than an adult would do under similar circumstances." In a paper "On some kinds of Albuminous and Purulent Urine in Children," read in the section of Diseases of Children at the meeting of the British Medical Association by Dr. Gee, 1883, he remarked that "stone in the kidney of a child sometimes seems to be latent, and to afford no signs beyond purulent urine. In cases of this kind I do not see how a diagnosis can be made. In other cases of stone in the kidney the diagnosis becomes possible,-when there are symptoms more or less like those of renal colic; when there are symptoms like stone in the bladder, and yet no stone can be discovered; or when the kidneys can be felt by deep pressure." Mr. R. W. Parker remarked at the same meeting that the absence of what in adults was considered one of the most characteristic signs, namely, renal pain, had often struck him. $\mathrm{He}$ mentioned the case of a girl who had been frequently subject to periodical attacks of hæmaturia and pyuria. She had no renal colic, and during the height of an attack only complained of a little uneasiness about the umbilicus. Thus it was difficult to say in which kidney the stone was, although doubtless a stone was the cause of the trouble. This patient was subsequently operated on, and the stone discovered.

Knowing as we do the great tendency in infants and children to the formation of urinary concretions, and the rarity of renal colic, the absence of the latter may be explained by two conditions :

(a) That in infants the calculi do not attain a sufficiently large size to become impacted, but as a rule are washed along the ureter without difficulty. 
(b) That the muscular tissue of the ureter is not sufficiently developed, as a rule, to cause such acute pain if a stone of a rather larger size than usual is passed.

It may also be remarked that the tissues of an infant being all lax and supple, a stone would be less likely to meet with the same relative opposition as in an adult, on account of the softness of the fibrous coat of the ureter, for we know that the fibrous structures in infants are especially soft and yielding, as is evidenced by the tendency to stretch in the various ligaments of the joints.

In adults we observe that after repeated attacks of renal colic, in some cases the sufferers do not endure continued pain from recurring attacks, and the explanation which is offered of this is that the muscular tissue atrophies, and thus the nerve supply being no longer present, pain ceases. The ureter is a muscular chanriel along which the calculus is forced by peristaltic action, and is composed of an external fibrous coat, a middle coat of plain muscular tissue, and a mucous lining. The mucous membrane is thin and smooth, and presents a few longitudinal folds when the ureter is open, and the nervous supply is derived from the inferior mesenteric, spermatic, and hypogastric plexuses. It is conceivable that the nerves supplying the ureter are not sufficiently developed to cause the acute pain met with in the adult by the passage of even a small stone from the kidney, and hence, in infants, the suffering is not so great; and that if, as in the cases brought forward, there is severe pain, it is exceptional, and may be due to a greater development of the nerve supply than usual, or to an inherited hypersensitiveness of the nervous system.

The subject of this paper is one which has afforded me great interest, more especially as I have met with the cases in illustration of it almost entirely in private practice. Neither at the Children's Hospital nor at the St. George's and St. James's Dispensary did I ever see a case, whilst I have only met with one in the out-patient department of the Grosvenor Hospital for Women and 
Children, where I have worked for years. This to me is almost conclusive that the affection is more rarely met with in the infants of the poor, and that the cause of this is due to the greater tendency to the inheritance of gouty conditions amongst the wealthier classes, the latter being more prone to the formation of uric acid, and to suffer from conditions classed under the name "gouty."

I have not gone into the matter of ætiology or treatment in this paper, for it would make it too long, and I have merely contented myself with describing the cases. There is, of course, great variety of opinion as to the best method of treatment in those liable to the frequent formation of uric acid. I may remark, however, that in the treatment of some boys whom I have had under my care, where every facility has been afforded for the greatest care and observation, and where the diet has been recorded every day, together with the conditions of the urine for weeks at a time, I have found that an ordinary plain mixed diet has agreed best, and furnished the most satisfactory result. In one obstinate case of a boy, where I tried dieting by cutting off nitrogenous food, and placing him on milk and fish, I considered that he was worse rather than better. He continually passed large quantities of free uric acid, which caused intense irritation, so that the bladder was frequently evacuated and gave him great inconvenience. After various dieting and drugs, I found that by giving tonics, ordering plain wholesome mixed diet with meat and vegetables, and allowing him to be in the open air as much as possible, he improved more rapidly than by any other treatment. It may be noted that if infants, on absolutely a milk diet, suffer from excessive formation of uric acid, children and adults are not likely to be cured of the same tendency by placing them on milk. In view of what has been said and written on dieting in cases of excessive formation of uric acid, this point is certainly worthy of being remembered. It may teach us also that as this affection is evidently due to defective chemistry in the body, we must en- 
deavour to find out to what that defect is due, and treat it accordingly.

That the subject of this paper has not previously been brought before the Society is due, in my opinion, to the fact that such cases as I have described, although undoubtedly rare, have been taken for severe cases of intestinal colic, which is, of course, extremely common.

The points to which I desire to draw attention are-

1st. That in these cases there was suddenness of onset of all the symptoms.

2nd. That there was nothing observable about the general condition of the children, and especially the urine, before the attack, to lead those in charge to seek medical advice.

3rd. That they had all the signs and symptoms of acute renal colic.

4th. That they all occurred in infants the offspring of gouty parents.

I believe I have proved in the foregoing paper that true renal colic occurs in infants ; and it is possible that if what I have written leads to more careful examination of infants suffering from acute pain, more numerous cases may be discovered, and that the symptoms of infants suffering as I have described will not be too hastily attributed to ordinary intestinal colic.

(For report of the discussion on this paper, see 'Proceedings of the Royal Medical and Chirurgical Society,' Third Series, vol. viii, p. 93.) 